\title{
دور المشروعات الصغيرة والمتوسطة في التنمية الاقتصادية
}

\section{يوسف سعد عيد الغريب(')،}

باحث دكتوراه -معهد البحوث والدراسات الأفريقية ودول حوض النيل - جامعة اسوان.

$$
\text { أ.د/ محمد عبد الوهاب ابو نحول }
$$

قسم الاقتصاد الزراعي - كلية الزراعة، جامعة أسيوط.

أ.د / رياض اسماعيل مصطفى رياض

قسم الاقتصاد الزراعي - كلية الزراعــة - جامعـــة العريـــش،

$$
\text { د. / محمود محمد عنبر }
$$

قسم الاقتصاد - كلية التجارة - جامعـــة أسوان

') وجب التتويه أن البحث جزء من رسالة الدكتوراه عنوانها " دور المشروعات الصغيرة والمتوسطة

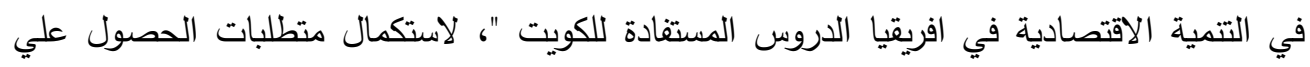
درجة الدكتوراه، معهد البحوث والدراسات الافريقية ودول حوض النيل فيل، جامعة أسون. 


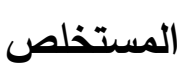

تهدف الدراسة إلي توضيح دور المشروعات الصغيرة والمتوسطة في تحقيق التتمية الاقتصادية، وتقوم الدراسة علي فرضية أن المشروعات الصغيرة والمتوسطة تحفز النمو الاقتصادي ومن ثم التتمية الاقتصادي، إذ تبين أن محددات النمو الاقتصادي تتمثل في التقدم التكنولوجي والابتكار والابحاث والتطوير ، وقد أثبت الدراسات أهمية المشروعات الصغيرة والمتوسطة في جذب الاستثمارات الاجنبية المباشرة والانفتاح علي حلقات تكنولوجية مهمة تؤدي إلي تحفيز النمو الاقتصادي، كما تسهم المشروعات الصغيرة في تحقيق التكامل الاقتصادي وخفض معدلات البطالة والفقر. الكلمات المفتاحية: المشروعات الصغيرة والتوسطة، التمية الاقتصادية

\section{Abstract}

The role of small and medium enterprises in economic development

The study aims to clarify the role of small and medium enterprises in achieving economic development, The study is based on the assumption that small and medium enterprises stimulate economic growth and then economic development, As it was found that the determinants of economic growth are 
technological progress, innovation, research and development, Studies have proven the importance of small and medium enterprises in attracting foreign direct investment and being open to important technological circles that lead to stimulating economic growth, Small enterprises also contribute to achieving economic integration and reducing unemployment and poverty rates.

\section{1 . 1 مقدمة}

ساهمت المشروعات الصغيرة والمتوسطة بدور هام في تحقيق التتمية الاقتصادية ودفع النشاط الاقتصادي سواء في الدول المتقدمة أو الدول التي تثهد تتمية مستدامة كدول شرق آسيا والصين والهند وكذلك الدول النامية، اذ ساهمت المشروعات الصغيرة في توفير العديد من فرص العمل للعاطلين، اضافة لمساهمتها في توفير السلع والخدمات الاساسية التي يحتاجها أفراد المجتمع لاسيما محدودي الدخل، كما ارتفعت القيمة المضافة للمشروعات الصغيرة والمتوسطة في العديد من الاقتصاديات التي أهتمت بتلك المشروعات، وساهمت المشروعات الصغيرة والمتوسطة في تتمية صادرات الدول المتقدمة، من خلال توفير السلع الوسيطة التي تحتاجها المشروعات الكبير وكانت تلجأ لنسبة كبيرة منها، وهو ما توضحه العديد من الدراسات السابقة،

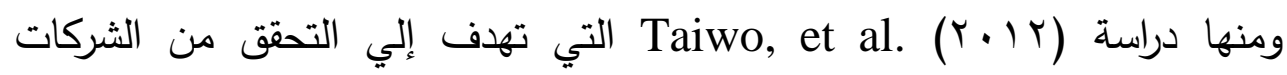


مجلة البحوث والداراسات الإفريقية ودول حوض النيل - جامعة أسون - المجلد (r) - العدد ( ) - يناير ( Y r م م)

الصغيرة والمتوسطة كأداة حقيقية في النمو الاقتصادي والتتمية، وتم استخدام طريقة المسح لجمع البيانات من . . شركة صغيرة ومتوسطة، مسئول ومدير من خمس حكومات محلية مختارة في نيجيريا وهي: حكومة إيجيبو الثمالية ويوا الجنوبية وساغامو وأوديدا وأوغون ووترسايد المحلية، وتم جمع البيانات باستخدام استبيان منظم وتحليلها مع العديد من الإحصاءات الوصفية لتحديد تصور أدوار الشركات الصغيرة والمتوسطة في نيجيريا، وتوصلت نتائج الدراسة أن القيود الأكثر شيوعًا التي تعرقل نمو الأعمال الصغيرة والمتوسطة في نيجيريا هي نقص الدعم المالي، وسوء الإدارة، والفساد، ونقص التدريب والخبرة، وضعف البنية التحتية، والأرباح غير الكافية، وانخفاض الطلب على المنتج وخدمات، ومن ثم، فهي توصي بأن تساعد الحكومة على وجه السرعة رواد الأعمال المحتملين في الحصول على التمويل والمعلومات الضرورية المتعلقة بفرص الأعمال والتكنولوجيا الحديثة والمواد الخام والسوق والمصنع والآلات التي من شأنها أن تمكنهم من خفض تكاليف التشغيل الخاصة بهم أكثر كفاءة لمواجهة منافسات السوق.

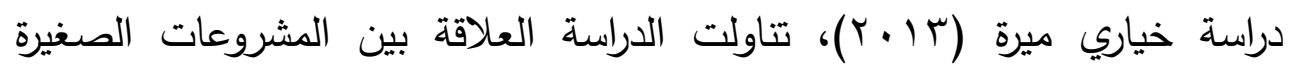
والمتوسطة والتتمة الاقتصادية، وباستخدام بيانات عن ولاية أم البواقي للفترة 


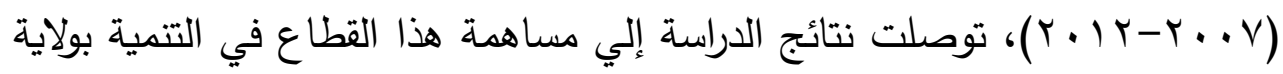
أم البواقي مازالت ضعيفة.

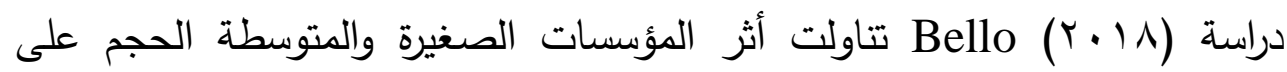
النمو الاقتصادي لنيجيريا باستخدام بيانات السلاسل الزمنية الممتدة بين عامي

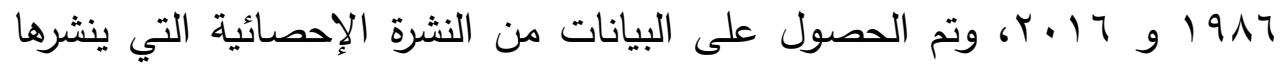
سنويًا البنك المركزي النيجيري (CBN). وباستخدام تحليل الانحدار لتفسير وتحليل البيانات التي تم جمعها للدراسة، توصلت نتائج الدراسة عن علاقة إيجابية وهامة بين الشركات الصغيرة والمتوسطة ونمو الإنتاج مما يشير إلى أن الشركات الصغيرة والمتوسطة الحجم في نيجيريا تقدم مساهمة إيجابية في تتمية الاقتصاد النيجيري، توصي الدراسة بضرورة قيام الحكومة بصياغة سياسة اقتصادية جديدة لتقييد الاستيراد المكثف للسلع الأجنبية خاصة تلك السلع التي يمكن أن تتتجها الشركات الصغيرة والمتوسطة محليًا من أجل حماية المنتجين المحليين من المنافسة مع الثركات الأجنبية. كما ينبغي بذل الجهود لضمان وجود مرافق بنية تحتية مناسبة للتشغيل السليم للشركات الصغيرة والمتوسطة في البلد.

كما تهدف الدراسة لتوضيح دور المشروعات الصغيرة والمتوسطة في تحقيق التتمية الاقتصادية، كما تقوم الدراسة علي فرضية أن المشروعات الصغيرة والمتوسطة تسهم 
مجلة البحوث والدراسات الإفريقية ودول حوض النيل - جامعة أسون - المجلد (r) - العدد (1) - يناير ( r · م م)

في دعم عمليات النمو الاقتصادي ومن ثم التتمية الاقتصادية، وفي هذا الاطار يقترح تقسيم الدراسة لنحو V أجزاء بالاضافة الي المقدمة، الجزء ا.Y يوضح مفهوم التتمية الاقتصادية، أما الجزء ا.ب يتناول شرح دور المشروعات الصغيرة والمتوسطة في دعم النمو الاقتصادي، الجزء ا.ء يتناول دور المشروعات الصغيرة والمتوسطة في الحد من البطالة والفقر، اما الجزء 10 يتتاول دور المشروعات الصغيرة والمتوسطة في تحقيق التكامل الاقتصادي، الجزء (.7 تحديات المشروعات الصغيرة والمتوسطة، ويتضمن الجزء الاخير الخلاصة.

\section{ا ب r مفهوم التنمية الاقتصادية}

لقد استخدم مصطلح النمو الاقتصادي والتتمية الاقتصادية بنفس المعني دون أي تفرقة بينهم في خمسينيات القرن العشرين، فالتتميه بالمفهوم التقليدي تعني تحقيق معدلات نمو مستدامه في نصيب الفرد من الاخل القومي، أي نمو الناتج المحلي الاجمالي بمعدل أسرع من معدل النمو في السكان، ثم تطور مفوم التتمية ليثمل احداث تغيرات هيكلية بجانب النمو الاقتصادي فمثلاً نلاحظ إنخفاض مساهمة الزراعه في كل من الإنتاج و العماله في المقابل زيادة نصيب كل من الصناعات

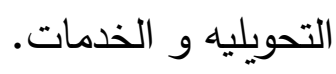


في الفترة الخمسينيات و الستينيات .901: . .9 أدي التركيز علي النمو الاقتصادي الي اتساع التفاوت في توزيع الدخول، مما أدي إلي ضرورة أن يشمل مفوم التنمية الاقتصادية خلال فترة السبعينيات من القرن العشرين: ارتفاع معدل النمو الاقتصادي+ احداث تغيرات هيكيلة+ تقليل التقاوت في توزيع الدخل القومي، والحد من الفقر وعدم المساواهو القضاء علي البطاله.

وجدير بالذكر أن "صن" Amartya sen's يري أن هناك خمس مصادر لتفاوت في توزيع الاخول هي، العوامل الشخصية، الاككانيات المتاحة للافراد، السن، النوع، الاعاقة، المرض...إلي اخره. عوامل بيئية، اختلاف درجة الحرارة وما تتطلبه منها مستلزمات للتكيف مع درجة الحرارة كثراء ملابس ثقيلة لمواجهة درجة الحرارة المنخفضة...إلي اخره العوامل الاجتماعية، راس المال الاجتماعي المتاح، درجة الامن والامان داخل المجتمع..إلي اخره، عدم العدالة في توزيع دخل الاسرة بين افرادها، تفضيل تعليم البنين علي البنات، وافر الرعاية الصحية للبنين علي البنات خاتتا في المجتمعات المتخلفة...إلي أخره.-Todaro and Smith, 2012:

يُلاحظ في فترة الثمنينات و التسعينيات ـ191 1: ـ99 حدثت تحولات في معدلات نمو الدخل القومي الإجمالي حتي وصل إلي السالب في كثير من الال الناميه و 
مجلة البحوث والدراسات الإفريقية ودول حوض النيل - جامعة أسون - المجلد (r) - العدد ( (1) - يناير ( r r م م)

الحكومات التي تواجه مشكله في تفاقم الدين الخارجي مما أضطرت هذه الدول

والحكومات علي إتباع البرامج الإقتصاديه والإجتماعيه المحدده من قبل المؤسسات

الاقتصادية الدولية، ترتب عليه الالتزام بأهداف التتمية تبنتها 91 1 دولة من الدول

الاعضاء في الامم المتحدة ما اطلق علي اعلان الالفية الثالثة في سبتمبر .... ب،

والذي يقوم علي هدف ضرورة تحسين مستوي معيشة الافراد في الدول النامية خلال

خمسة وعشرون عاماً- من سنة . 199 لسنة 10 ـ. (امنية عز الدين وسمية عبد

$$
\text { المولي، · ( • ب: هب) }
$$

جدول (1)

أهداف التنمية للألفية الثالثة

\begin{tabular}{|c|c|c|}
\hline مضمون هذا الهدف & & 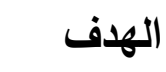 \\
\hline تخفيض عدد الافراد الذين يعيشون تحت خط الفقر & علي الفقر & والمجاعة \\
\hline
\end{tabular}




\begin{tabular}{|c|c|}
\hline 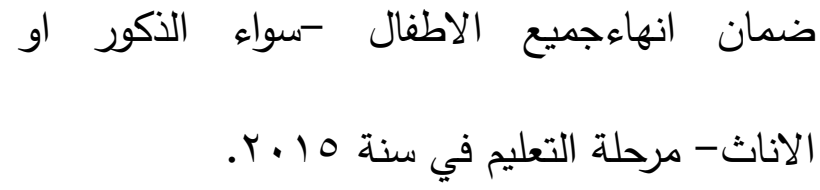 & الابتدائية \\
\hline 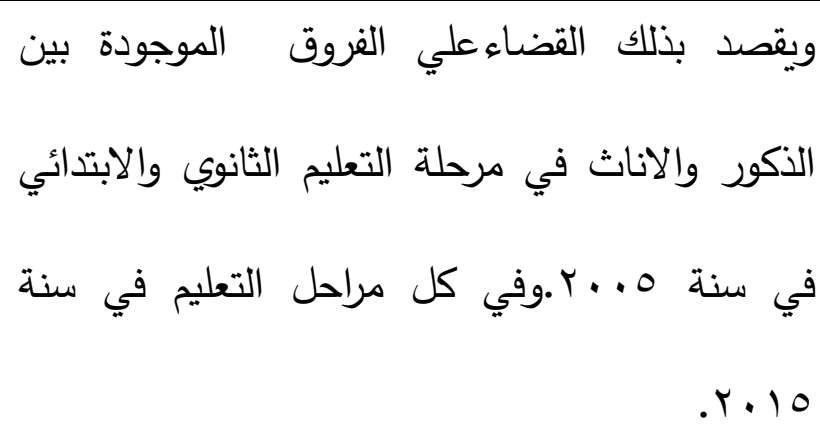 & 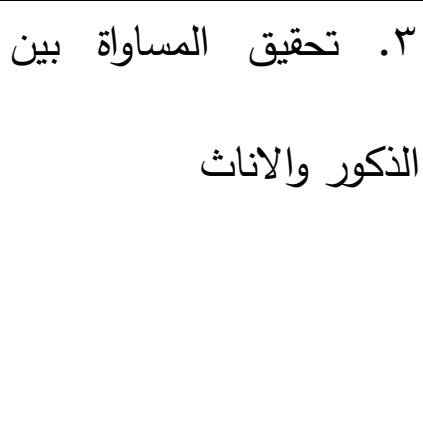 \\
\hline وهنا تهدف اهداف الالفية الثالثة الي تخفيض معدل & ع ـتخفيض معدل الوفيات \\
\hline يعني هذا الهدف تخفيض معدل وفيات الامهات & الحومل صحة الامهات \\
\hline وذلك من خلال تقليل انتشر كل من مرض الايدز & الخطاربة \\
\hline ذلك من خلال اخذفهوم التتمية المستدامة في & V Vمان حماية البيئة \\
\hline
\end{tabular}


مجلة البحوث والدراسات الإفريقية ودول حوض النيل - جامعة أسون - المجلد (r) - العدد ( (1) - يناير ( r r م م)

الاعتبار عن تحديد برامج وسياسات التتمية

وتخفيض عدد الافراد الذين لايحصلون علي مياه

نظيفة صالحة للشرب الي النصف.

الغاء الديون الخارجية للدول الفقيرة.

^دم المشاركة في

توفير العمل الملائم لقوة العمل خاصتنا الشباب.

التنمية

الاتفاق مع شركات لتوفير الادوية الضرورية للعالم

النامي.

تقديم الدول المتقدمة والمنظمات الدولية مساعدات

للعالم النامي بدف تخفيض الفقر .

يتضح مما سبق ان اهداف التنمية للالفية الثالثة التي حددت في اطار الامم

المتحدة تتمثل في هدف اساسي وهو تخفيض مستوي الفقر ورفع مستوي معيشة

الافراد.ومن ثم فان مفهوم التتمية الاقتصادية يجب أن يتضمن هدفي تخفيف مستوي

الفقر ورفع مستوي معيشة الافراد في الدول النامية.

ومن كل ما سبق يمكن القول أن مفهوم التنمية الاقتصادية مفهوما واسعاً يشمل جوانب متعددة فالتتمية لايجب أن تقتصر علي النمو الاقتصادي ولكنها أوسع مدي 
من ذلك حيث تثمل ضرورة أحداث تغييرات هيكلية رفع مستوي معيشة الافراد عن طريق القضاء علي الفقر وتخفيف التفاوت في توزيع الدخل وتوفير الخدات الاساسية وفرص العمل، هذا بجانب المحافظة علي البيئة حتي يعيش الافراد في محيط صحي جيد، وحديثا تثمل التتمية الاقتصادية تحقيق أهداف التتمية المستدامة. جدير بالذكر أن مفهوم التمية الاقتصادية بالجوانب السياسية من خلال تثجيع الافراد علي المشاركة السياسية والعمل علي تتمية الثر بالانتماء والثعور بالامان، والمقصود بالمشاركة السياسية اي مشاركة الافراد -بصورة مباشرة او غير مباشرةفي منافثة الاهدف التي تسعي التتمية الاقتصادية الي تحقيقها خلال فترة زمنية معينة مما يعني المشاركة في صنع القرار. ولاثك ان شعور الافراد (العاملين وملاكك عناصر الانتاج) بالمشاركة في تحديد أهداف التنمية سيزيد من رغبتهم في تنفيذ هذه الاهداف، ويخلص من ذلك أن الفرد ينبغي ان يكون الهدف الاساسي والنهائي للتمية الاقتصادية لانه صانعها ومن ثم فمن حقه جني ثمارها، بمعني ان تعود عليه بالنفع المتمثل في حقه أن يحيا حياة كريمة. 1 ا دور المشروعات الصغيرة والمتوسطة في دعم النمو الاقتصادي 


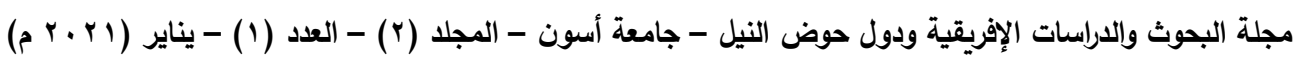

يُعد النمو الاقتصادي احد المكونات الرئيسية للتنمية الاقتصادية، فأرتفاع النمو الاقتصادي يؤدي الي ارتفاع معدلات التتمية الاقتصادية، ويتطلب رفع معدل النمو الاقتصادي في اقتصاد ما معرفة محدداته والتي يتم استتتاجها من نظريات النمو الاقتصادي، الامر الذي يساعد في استتباط دور المشروعات الصغيرة والمتوسطة في دعم النمو الاقتصادي، وسوف يتم توضيح نموذج سولو ونظريات النمو الحديثة، والثورة النيوكلاسيكية المعاكسة كنظريات مفسرة لمحدات النمو الاقتصادي ومحاولة استنباط منها دور المشروعات الصغيرة والمتوسطة في تحفيز النمو الاقتصادي ومن ثم التمية الاقتصادية.

بالنسبة لنموذج سولو Solow يعتبر من أهم النماذج الكلاسيكية الحديثة في النمو ويقوم نموذج سولو علي توسيع أطار نموذج هارود دومار عن طريق ادخال عاملين إلي راس المال، أو الادخار كمحدات للنمو هما العمل والتكنولوجيا( المدخلات) تساوي نسبة الزيادة في الناتج ( للمخرجات)، فقد افترض سولو تناقص الغلة لكل من عنصر العمل وراس المال منفصلين وثبات الغلة بالنسبة لهما جميعاً، أما التطور التكنولوجي فقد أخذه سولو علي انه عامل متبقي يشرح النمو في الاجل الطويل، كما

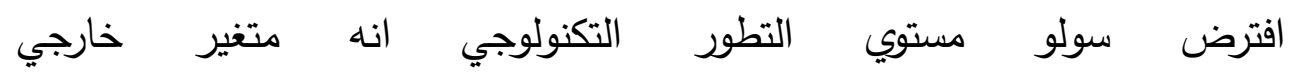
(Todaro and Smith, 2012:126).Exogenous 
لقد استخدم سولو دالة كوب - دوجلاس في شرحه النموذج الكلاسيكي الحديث في النمو معبراً عن ذلك بالصيغة التالية:

$$
\mathrm{Y}=\mathrm{A} e^{u t} k^{\alpha} l^{1-\alpha}
$$

حيث ان Y تثير الي الناتج المحلي الاجمالي،K تثير الي راس المال المادي، وراس المال البشري، L تثير الي العمل الغير ماهر، A تشير الي ثابت ويمثل مستوي التكنولوجيا الاساسي، e تشير الي معدل النمو للتكنولوجيا وهوثابت يفسر Todaro and ) النمو في الاجل الطويل وهو ايضاً يعرف بمتبقي سولو .(Smith,2012:129

طبقاً لسولو فإن ه،( ه-1 ) كلاهما اقل من الواحد الصحيح وهكذا فإن نمو الناتج يعتمد علي واحد أو اكثر من الثلاث عوامل في المعادلة الدذكورة وهي العمل وراس المال والتطور التكنولوجي، وقد أثبت الدراسات أهمية المشروعات الصغيرة والمتوسطة في جذب الاستثارات الاجنبية المباشرة والانفتاح علي حلقات تكنولوجية مهمة تؤدي إلي تحفيز النمو الاقتصادي، ففي تقرير للاونتكاد توصل من مسح ميداني ودراسة حالات عديدة لبلدان النمور الاسيوية، توصلت إلي المشاريع الصغيرة والمتوسطة لها قدرة علي استقطاب نسبة مهمة من الاستثارات الاجنبية المباشرة، وتسببت في رفع حصتها من الاستثمارات الاجنبية المباشرة لأكثر من نحو (1\% من 


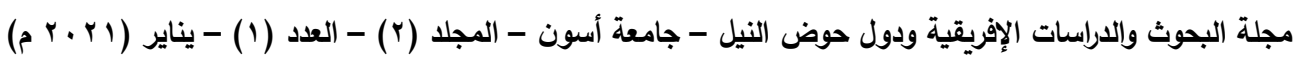

الاستثمارات الاجنبية المباشرة رغم الازمة المالية عام 99 1 التي أدت الي خسائر كبير بها، بسبب قدرة المشروعات الصغيرة والمتوسطة علي الدخول في مشاريع مشتركة مع الاجانب. (UNCTAD, 1998: 4)

أما نظرية النمو الحديثة تري أن العوامل الرئيسية المولدة للنمو والتي اوردها روادها اتجاه النمو الذاتي في أربعة مصادر راس المال المادي أوالمعرفة(رومر)، راس المال البشري(لوكاس)، راس المال التكنولوجي(رومر-اجيون وهويت- هيلمان وجروسمان)، راس المال العام(باور).

أ. نموذج رومر الاول(919 (1) بني رومر نموذجه الاول علي عدة فروض: منها أن المعرفة تمثل الثكل الرئيسي لراس المال، وهي نتاج الابحاث التي تتم في مجال الفن الانتاجي، والتي تظهر تتاقص عائد النطاق( اي انه في ضوء رصيد معين للمعرفة في أي لحظة زمنية معينة فإن مضاعفة المدخلات المستخدمة في إنتاج الابحاث لن يترتب عليه مضاعفة مقدار المعرفة الجديدة المنتجة)، ويتمثل التقدم التكنولوجي في انتاج انواع جديدة من السلع الراسمالية، استبعاد فرض تتاقص الناتج الحدي لراس المالي أي أن الناتج الحدي لراس المال اما ان يكون ثابت أومتزايد وليس متتاقص.(Romer,1986: 1002-1037). 
ووفقا لرومر فرأس المال الذاتي(هو المحرك الاساسي لعملية النمو الاقتصادي الذاتي)، وهو مخزن المعرفة المتولدة من راس المال المادي التي تتتج تلقائياً من الخبرة المكتبة من الانتاج. كما توجد ثلاث حالات للنمو: الاولي، ان يكون معامل مرونة انتاج المعرفة الكلية(أي المعرفة الخاصة والجماعية) أقل من الواحد الصحيح، وهنا لاتوجد أي آثار خارجية ايجابية لراس المال ويتوقف النمو في الاجل الطويل، الثانية اذا كان معامل مرونة المعرفة الكلية تعادل الواحد صحيح فإن النمو يتم بمعدل ثابت(نمو متوازن)، الثالثة أن يكون معامل مرونة انتاج المعرفة الكلية اكبر من الواحد الصحيح فإن معدل النمو سوف يكون في تزايد مستمر (يسري بطرس $\cdot(\varepsilon r-\varepsilon r: r \cdot 1)$

ب. نموذج لوكاس، قد أهتم نموذج لوكاس براس المال البشري كمرك للنمو، وراس المال البشري هو مخزن المعارف المكتسبة من خلال الاعداد والتأهيل والتي تزيد من فاعليتهم الانتاجية وهي لاتتصرف فقط الي مستوي الكفاءات وانما كذلك الي حالة

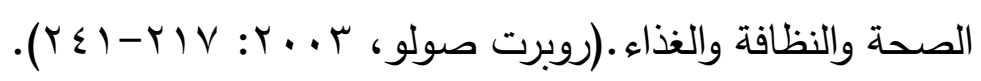

وهنا يلاحظ ان راس المال البشري يمثل ملكية خاصة للفرد الذي يحمله، فالمعرفة تُعد صفة للفرد الذي يحوزها، والاصل في عوائد تراكم راس المال البشري انها خاصة، بينما راس المال التكنولوجي هي مالاً عاماً بحيث لايتطلب أن يبذل 


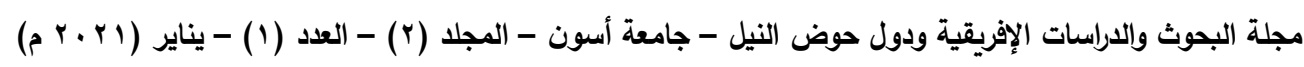

المتخصصين ذات الجهود التي أجريت براس المال التكنولوجي والعوائد الخاصة براس المال التكنولوجي هي في المقام الاول عمومية.

وأكد لوكاس علي أن راس المال البشري يتم في المدارس ومراكز التدريب ويتم ايضاً بدرجة لاتقل أهمية اثناء انتاج السلع والقيام بالتبادل التجاري، كما أكد لوكاس علي أهمية تراكم راس المال البشري وهو أهم عامل في تحقيق النمو الاقتصادي وأن تراكم راس المال البشري يلعب دوراً هاماً في عملية النمو. ج. نموذج رومر الثاني(• 99 ())، وفقاً لنموذج رومر الثاني فان الابتكارات تدفع الي تطبيق اساليب انتاجية جديدة وتتسم بالطابع الاحتكاري، ومن ثم تحقق فائدة اضافية فبسبب قصر حق استعمالها علي مشتري البراءة الذي ليس هو المخترع يمكنه ان يطلب سعراً أعلي من التكلفة الحدية، وعلي الشركات ان تسعي لاكتساب المعرفة حتي يمكنها انتاج سلع جدية ( نظام براءات الاختراع). (-Romer,1990: 98 130). ومن ثم فانتاج المعارف يحقق نوعين من الايرادات وهي الايرادات الخاصة( ثمن بيع براءات الاختراع للمكتشف)، وبراءات جماعية وهي اعلي من الايرادات الخاصة لما تحققه من اثار خارجية ومن هذا المنطق فإن المساعدات التي تقدمها الدول للبحوث تعتبر فعالة نظراً لانها تساهم في زيادة معدل النمو الاقتصادي. 
وبالتالي يري رومر ان الدخزون الاجمالي للمعارف المتمثل في عدد من الابتكارات الاجمالية الماضية، لها وفر خارجي ايجابي علي البحوث اللاحقة وبالتالي فان الوفورات الايجابية للبحث التي تتنشر في عموم الاقتصاد تؤدي الي ان يكون معدل النمو المتوازن ادني من المعدل الامثل. - المثل

د. نموذج أجيون وهويت 1991، يوضح لنا ان تحقيق معدل نمو متوازن وثابت يتطلب تخصيص حجم ثابت وكاف من الموارد للبحث والتطوير، وهنا تكون دالة الانتاج متزايدة للاستكثافات، بينما في حالة تخصيص حجم ضعيف من الموارد للبحث والتطوير تكون هناك علاقة عكسية مع التجديد، حيث يصبح معدل النمو غير حقيقي، بينما في حالة تحقيق وضع سط بين الوضعين السابقين اي مرحلة تعاقب مرحلة التخصيص القوي والضعيف من الموارد للبحث والتطوير يؤدي الي دخول الاقتصاد في شبه دورة النمو.

والتقدم الفني وفقاً لنموذج اجيون وهويت يتمثل في اختراع سلع وسيطة جديدة تحل محل تلك التي كانت موجودة من قبل مما يترتب عليه اثرين، اما نقص تكاليف

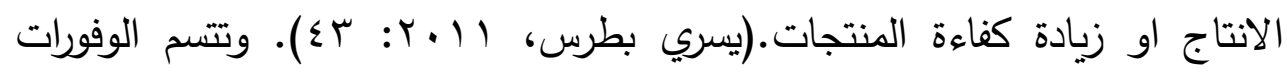
الخارجية وفقاً لنموذج أجيون وهويت بصفتين: الاولي، انها مؤقتة أي تتوقف بمجرد ظهور تجديد تلو اخر، الثانية، انها تحتوي في طياتها وفراً سلبياً، فالتجديد يؤدي الي 
مجلة البحوث والدراسات الإفريقية ودول حوض النيل - جامعة أسون - المجلد (r) - العدد (1) - يناير ( r · م م)

هدم الاكتشافات الجديدة، ويعتمد حجم الموارد المخصصة للبحث في النموذج سلبياً علي معدل الفائدة وايجابياً علي مستوي الاكتشافات والتكنولوجية المطبقة وعدد العاملين الذي يمكن ان يوظفوا في قطاع البحث والتطوير ويلاحظ ان مستوي النمو المتوازن أقل من مستوي النمو الأمثل في هذا النموذج. فانه مع بدء ظهور اكتشاف جديد تزول الوفورات الخارجية الايجابية لتحل محلها وفورات خارجية سلبية. بصفة عامة، يمكن التعبير عن اغلب نظريات النمو الداخلي باستخدام المعادلة التالية:Y=AK حيث ان: A تعبر عن اي عامل يؤثر في التكنولوجيا، K تتضمن كل من راس المال البشري والمادي، Yالناتج.(ميشل تودارو،999 (1: 100) يبدو واضحاً من هذه المعادلة عدم وجود ما يعرف بتناقص العائد علي راس المال إذا أن الاستثمارات في راس المال المادي والبشري تولا اقتصاديات خارجة (الوفورات)وتحسنات معدل الانتاجية التي تتعدي المكاسب الخاصة بكمية كافية لمقابلة تناقص العوائد، فهناك امكانية وفقاً لنموذج النمو الداخل في اي الاستثمارات المولدة للاقتصادات الخارجة تجعل م في معادلة سولو تساوي الواحد صحيح ومعادلة سولو أو دالة الانتاج عند سولو كالتالي:

(2) $\mathrm{Y}=\mathrm{A} e^{u t} k^{\alpha} l^{1-\alpha}$ 
حيث ان Y تثير الي الناتج المحلي الاجمالي،K تثير الي راس المال المادي Physical ، وراس المال البشري L ، Human Capital تثير الي العمل الغير ماهر ، A تثير الي ثابت ويمثل مستوي التكنولوجيا الاساسي، e تثير الي معدل النمو للتكنولوجيا وهوثابت يفسر النمو في الاجل الطويل وهو ايضاً يعرف بمتبقي

$$
\begin{gathered}
\text { سولو وعندما نعوض } \begin{array}{l}
\text { Y }=\mathrm{A} e^{u t} k^{\alpha} l^{1-1} \\
\mathrm{Y}=\mathrm{A} e^{u t} k^{1} l^{0} \\
\mathrm{Y}=\mathrm{A} e^{u t} k *(1)
\end{array} \\
\mathrm{Y}=\mathrm{A} e^{u t} k
\end{gathered}
$$

تعرف المعادلة (ع) بمعادلة سولو وتصبح النتيجة الصافية للنمو المتواصل في الاجل الطويل نتيجة زيادة العائد بالنسبة للحجم لذلك وهكذا يمكن القول ان نظرية النمو الحديثة تعيد التاكيد علي أهمية المدخرات واستثمارات راس المال البشري لتحقيق نمو سريع في دول العالم النامي.

واذا كانت محددات النمو الاقتصادي وفقا لنظريات النمو الاقتصادي الحديث تتمثل في الاستثمار في البشر والاستثمار في قطاع البحث والتطوير فإن الشروعات 


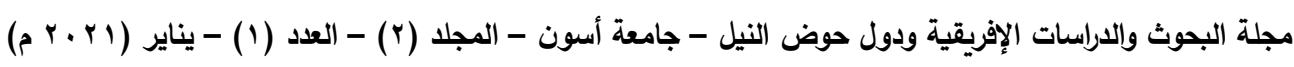

الصغيرة والمتوسطة تساهم بنحو ـ٪ من كل الابداعات والابتكارات الجديدة في

(Thorsten, et al., 2003:22) السوق الامريكي

الثورة النيوكلاسيكية المعاكسة حدث في عقد الثمانينيات من القرن العشرين أن صعدت القوي السياسية المحافظة الي الحكم في كل من الولايات المتحدة الامريكية، وكندا، انجلترا، وبريطانيا والمانيا الغربية وقبل اعادة توحيد المانيا؛ مما ادي الي ظهور الثورة النيوكلاسيكية المعاكسة في النظريات والسياسات الاقتصادية ولقد Harry Johnson, Deep ظهرت مدرسة الثورة النيو كلاسيكية المعاكسة بزعامة Lai, Lord Peter Bauer, Bela Balassa. ان التخلف الاقتصادي نتج عن سوء تخصيص الموارد بسبب السياسات السعرية الخاطئة والتتخل المنوط في النشاط الاقتصادي من جانب حكومات دول العالم الثالث كما ان تدخل الدولة في النشاط الاقتصادي هو الذي يؤدي الي إبطاء عملية النمو الاقتصادي.

فوفقاً لمنهج الاختيار العام ويطلق عليه منهج الاقتصاد السياسي الجديد - كاحد المناهج المعبرة عن نماذح الثورة النيوكلاسيكية الثلاثة- فان السياسيون يستخدما موارد الحكومة في تعزيز ما يتمتعون به من نفوذ وسلطة، كذلك يستخدم موظفو الحكومة ومسئولوها وظائفهم للحصول علي الرشاوي من المواطنين الباحثين عن 
المكاسب الريعية او الراغبين في تمتع مشروعاتهم بصورة الحماية المختلفة. واخيراً، تستخدم الحكومات سطوتها لمصادرة الممتلكات الخاصة من الافراد وعلي ذلك تكون نتيجة الصافية لكل من الممارسات هي سوء تخصيص الموارد بالاضافة للانخفاض العام في الحريات الفردية. (Todaro and Smith,2012:126-127).

يري الليبراليون الجدد أن السماح بانتعاش الاسواق الحرة، وخصخصة المشروعات المملوكة للدولة وتثجيع حرية التجارة والتصدير ، والترحيب بالمستثمرين الاجانب من الدول الدتقدمة، وتقليص صور التذخل الحكومي والاختلالات السعرية سواء في عوامل الانتاج او السلع او اسواق راس المال، ومن المتوقع ان يؤدي كل ذلك الي زيادة الكفاءة الاقتصادية والنمو الاقتصادي، وعلي ذلك، يمكن استتاج أهمية المشروعات الصغيرة والمتوسطة في رفع معد النمو الاقتصادي أو التخلص من عوائق النمو الاقتصادي الناتجة عن الفساد البيوقراطي في المشروعات العملاقة، كما يمكن القول ووفقا لفكر الثورة النيو كلاسيكية فإن ما تحتاجه الدول النامية ليس مجرد اصلاح النظام الاقتصادي الدولي فحسب وانما عملية إعادة هيكلة للتخلص من ظاهرة الثنائية التي تعاني منها، وزيادة المعونات الاجنبية، ومحاولة السيطرة علي معدلات النمو السكاني ونظام تخطيط مركزي يكون اكثر فعالية. 


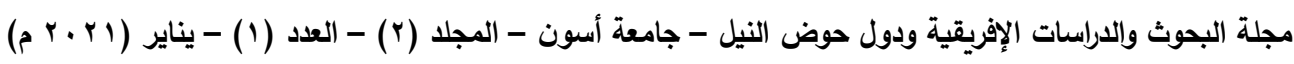

إن ما تحتاجه هذه الدول - ببساطة شديدة- هو تشجيع وجود أسواق حرة وتطبيق مبدأ"إتركه يعمل Laissez Faire " من خلال حكومات تقوم بتيسيرات تسمح لقوي السوق بالعمل، بحيث تكون اسعار السوق مرشداً لعملية تخصيص الموارد، وتثجيع النمو الاقتصادي. وقد اشار هؤلاء المؤيدون للاسواق الحرة الي قصص النجاح التي تحقت في دول مثل كوريا الجنوبية وسنغافورة كامثلة للاسواق الحرة، والي قصص الفشل التي تحقتت في الاقتصاديات القائمة علي الدخل الحكومي في قيادة امريكا اللاتينية، وجدير بالذكر ان انصار مدرسة الثورة النيوكلاسيكية علي اقوي مؤستتين ماليتين في العالم هما البنك الدولي وصندوق النقد الدولي وقدتزامن ذلك مع حدوث ت ILO Iاكل في نفوذبعض المنظمات والمؤسسات الدولية مثل منظمة العمل الدولية برنامج الامم المتحدة للتمية UNDP ، ومؤتمر الامم المتحدة للتجارة والتتمية UNCIAD ، وهي تعكس وجهة نظر الدول الاقل تقدماً، وهو بشكل تحدياً لاصحاب حجة التدخل في النشاط الاقتصادي وانصار نظرية التبعية. ( Todaro (and Smith,2012:1126-217).ويتبين مما سبق ان الحكومات ذات الحجم الضئيل جداً هي افضل الحكومات.

وقد أثبت الدراسات وجود علاقة طردية ين ارتفاع عدد المشروعات الصغيرة والمتوسطة وبين التطور المؤسسي في العديد من البلدان التي شملتها الدراسات، اذ 
تسهمالمشروعات الصغيرة والمتوسطة في تنويع الهيكل الاقتصادي كما تساعد في تغيير الهيكل السوقي من خلال تعزيز القدرة التصديرية وتوسيع قاعدة الانتاج وتوطين التثنية الحديثة. (Waite, 1973: 82)

ـ ـ دور المشروعات الصغيرة والمتوسطة في خفض معدلات البطالة

والفقر

تتميز المشروعات الصغيرة والمتوسطة بقدرتها علي الحد من البطالة، إذ تسهح تلك المشروعات في استعاب العمالة الغير ماهيرة أو النصف ماهرة التي تثكل نسبة مهمة في البلدان النامية، وتتميز المشروعات الصغيرة عن المشروعات الكبيرة في القدرة علي خلق فرصة بتكاليف منخفضة نسبياً أي بالنسبة لتكلفة فرصةالعمل التي توفرها المشروعات الكبري، بالاضافة الي قدرة المشروعات الصغيرة والمتوسطة علي التركزفي المناطق النائية الامر الذي يمكنها من الحد من ظاهرة البطالة الريفية المتراكمة، كما أن المشروعات الصغيرة والمتوسطة يمكن اعتبارها آلية فعالة لمكافحة الفقر ، لاسيما في الاقاليم النائية الاقل حظاً في النمو أو الأكثر احتياجاً للتمية، من خلال اتاحة التمويل لصغار المستثرين والمرأة المعلية، وجدير بالذكر أدت أزمة البطالة في السبعينيات إلي الاهتمام بالمشاريع الصغيرة والمتوسطة للحد من تلك 
مجلة البحوث والدراسات الإفريقية ودول حوض النيل - جامعة أسون - المجلد (r) - العدد (1) - يناير ( r · م م)

البطالة لاسيما بطالة الخريجين، كما أنه في الاونة الاخيرة بدأ الاهتمام بشروعات

ريادة الاعمال وتتميتها كوسلية لامتصاص بطالة الشباب المرتفع في أغلب البلدان

العربية.

ا.0 دور المشروعات الصغيرة والمتوسطة في تحقيق التكامل

الاقتصادي

تتسم المشروعات الصغيرة والمتوسطة بالمرونة وقدرتها علي تحمل تكاليف الانتاج، والرقابة، والامكانيات، وهو أدي الي قيام المشروعات الكبيرة بالاعتماد علي المشروعات الصغيرة والمتوسطة في انتاج العديد من احتياجاتها السلعية. (نثأت

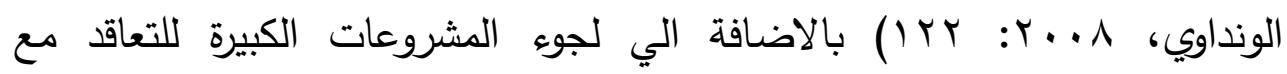
المشروعات الصغيرة والمتوسطة للحصول علي بعض الخدمات او السلع الوسيطة، بما يعزز حالة التكامل الصناعي أو ما يسمي في الاقتصاد التعاقد بالباطن، بالاضافة إلي أن المشروعات الصغيرة تمثل نواة اساسية للمشروعات الكبري فأغلب المشروعات الكبري بدأت من مشروعات صغيرة ومتوسطة، كما يؤدي تتمية المشروعات الصغيرة والمتوسطة إلي استحداث منتجات جديدة واستيعاب جزء من 
ناتج المشروعات الكبيرة الامر الذي يؤدي الي تقليل الفاقد أو الهدر بالاضافة إلي

$$
\text { الحد من الاستيراد. }
$$

\section{1.}

تواجه المشروعات الصغيرة والمتوسطة العديد من التحديات، وهذه التحديات أو المعوقات التي تواجه المشروعات الصغيرة والمتوسطة تختلف في طبيعتها عن تلك المشكلات التي تواجه المشروعات الكبيرة، اذ أن التحديات التي تواجه المشروعات الصغيرة والمتوسطة تتسم بأنها متداخلة من حيث أسبابها ونتائجها، وتتمثل المشكلات التي تواجه المشروعات الصغيرة والمتوسطة في، ضعف مساهمة المشروعات الصغيرة والمتوسطة في تكوين الناتج العالمي، والعديد من المشكلات الادارية، والتسويقية، والثقافية.

تشير الاحصائيات الدولية الي انخفاض مساهمة المشروعات الصغيرة والمتوسطة في تكوين الناتج المحلي الاجمالي، فالمشروعات الصغيرة والمتوسطة التي تستوعب نحو ثلثي اجمالي العمالة في البلدان النامية والمتقدمة معاً، تساهم في تكوين الناتج الكلي للدول النامية والمتقدمة بنسب قدرت بنحوهب\% ونحو 0\% فقط، علي الترتيب وهي نسبة منخفضة ايضاً، وقد يرجع هذا لإنخفاض انتاجية العاملين في القطاعات 


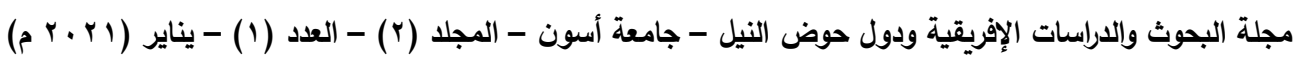

الصغيرة والمتوسطة نسبياً أي بالنسبة للمشروعات الكبيرة فنحو • ٪\%ن الثركات الصغيرة والمتوسطة أقل إنتاجية من المشروعات الكبيرة. ) World Trade (Report, 2016:13

المعوقات التمويلية، حيث تختلف نسبة القروض المخصصة للمشروعات الصغيرة من دولة لأخري، وإن كانت منخفضة علي اية حال، بالاضافة الي مواجهة بعض المشروعات الصغيرة والمتوسطة صعوبات عند الحصول علي التمويل اللازم لها بسبب عدم ملائمة المعايير في المصارف لطبيعة هذه المشروعات، بالاضافة الي الصعوبات المالية الذاتية من حيث عدم انتظام التدفقات المالية الداخلة الذي يؤدي الي رفع درجة مخاطر الائتمان الممنوح لها.

وتسعي الحكومات في الاونة الاخيرة لمحاولة دعم وتطوير قطاع المشروعات الصغيرة والمتوسطة، فعلي سبيل المثال - لا الحصر - سعي البنك المركزي المصري الي دعم تلك المشروعات من خلال اصدار عدة قرارات يترتب عليها تخصيص نحو . . . مليار جنيه لدعم مشروعات هذا القطاع خلال ع سنوات تتتهي في .Y.r. والزام البنوك بتخصيص نحو .r\% من محفظة القروض لديها مع تأسيس ادارة مخصصة لتمويل وعدم تلك مشروعات تلك القطاع، بالاضافة لتقديم برامج خصيصاً لتأهيل العاملين بالبنوك من خلال المعهد المصرفي، اصدار التعريف الموحد 
لمشروعات هذا القطاع في ديسبر 10 بـ، مع اجراء تعديل علي التعريف في مارس VI V • ليتوافق مع المتغيرات الاقتصادية المصاحبة لسياسات التعويم الحر لمعدل الصرف الاجنبي.

كما يعاني قطاع المشروعات متناهية الصغري والمتوسطة في الدول النامية من العوائق الثقافية كالمفاهيم التي لازالت سائدة في المجتمات النامية عن أن الوظيفة الحكومية هي الملأذ المثالي من حيث الأمان والاحترام الاجتماعي، بالاضافة الي العوائق التعليمية، فالمناهج العلمية لازالت لم تمد اصحاب الاعمال الواعدين بالمهارات الادارية والفنية اللازمة لممارسة الاعمال الحرة، العوائق المؤسسة كالاجراءات البيوقراطية، ومخاطر الاقتصاد الكلي كمعدلات التضخم المرتفعة، وأثر سياسات السوق الحر كسياسات التوعيم الحر لمعدل الصرف الاجنبي علي هذه المشروعات، بالاضافة لغياب الخبرة الفنية (حسين الاسرج، 7 ( ب: 99).

أما المعوقات التسويقية، منها، تفضيل المستهلك للمنتجات الاجنبية لمجرد تقليده للنمط الغربي في الاستهلاك، او ضعف منافسة المنتجات المحلية للمنتجات الاوروبية، أو ضعف الاهتمام بالبحوث التسويقية، ونقص المعلومات عن السوق، وبالنسبة للمعوقات الادارية فمنها، اعتماد نمط المدير المالك غير المحترف، وغياب 
مجلة البحوث والدراسات الإفريقية ودول حوض النيل - جامعة أسون - المجلد (r) - العدد (1) - يناير ( r · م م)

الكوارد المدربة أوالمؤهلة اداريا في تلك المشروعات. (كفاح أبو ناجي، عـ ـ ب: بr-

\section{(الخلاصة ا}

تهدف الدراسة إلي توضيح دور المشروعات الصغيرة والمتوسطة في تحقيق التتمية الاقتصادية، وفي هذا الاطار تم تقسيم الدراسة لنحو سبعة أجزاء بالإضافة إلي المقدمة، الجزء الثاني وضح مفهوم التتمية الاقتصادية، أما الجزء الثالث تتاول شرح لدور المشروعات الصغيرة والمتوسطة في دعم النمو الاقتصادي، الجزء الرابع تناول توضيح لدور المشروعات الصغيرة والمتوسطة في خفض معدلات البطالة والفقر، والجزء الخامس تضمن شرح لدور المشروعات الصغيرة والمتوسطة في تحقيق التكامل الاقتصادي، الجزء السادس وضح تحديات المشروعات الصغيرة والمتوسطة، ويتناول الجزء الاخير الخلاصة.

أما الجزء الثاني الذي تضمن توضيح لمفهوم التتمية الاقتصادية، توصل إلي أن التتمية الاقتصادية مفهوما واسعاً يشمل جوانب متعددة فالتمية لايجب أن تقتصر علي النمو الاقتصادي ولكنها أوسع مدي من ذلك حيث تثمل ضرورة أحداث تغييرات هيكلية رفع مستوي معيشة الافراد عن طريق القضاء علي الفقر وتخفيف 
التفاوت في توزيع الدخل وتوفير الخدمات الاساسية وفرص العمل، هذا بجانب المحافظة علي البيئة حتي يعيش الافراد في محيط صحي جيد، وحديثا تثمل التتمية الاقتصادية تحقيق أهداف التتمية المستدامة. وبالنسبة للجزء الثالث الذي يشرح دور المشروعات الصغيرة والمتوسطة في دعم النمو الاقتصادي فقد اشتمل هذا الجزء علي توضيح لنموذج سولو في النمو الاقتصادي، ونماذج النمو الحديثة، والثورة النيو كلاسيكية الحديثة حيث تبين أن محددات النمو الاقتصادي تتمثل في التقدم التكنولوجي والابتكار والابحاث والتطوير، وقد أثبت الدراسات أهمية المشروعات الصغيرة والمتوسطة في جذب الاستثمارات الاجنبية المباشرة والانفتاح علي حلقات تكنولوجية مهمة تؤدي إلي تحفيز النمو

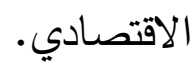

اما الجزء الرابع فتضمن توضيح دور المشروعات الصغيرة والمتوسطة في خفض معدلات البطالة والفقر ، كما تناول الجزء الخامس لدور المشروعات الصغيرة والمتوسطة في تحقيق التكامل الاقتصادي، حيث أن المشروعات الصغيرة تسهم في تحقيق التكامل الاقتصادي وخفض معدلات البطالة والفقر. 
مجلة البحوث والدراسات الإفريقية ودول حوض النيل - جامعة أسون - المجلد (r) - العدد ( (1) - يناير ( r r م م)

وتضمن الجزء السادس شرح لتحديات المشروعات الصغيرة والمتوسطة، اذ توجد

العديد من التحديات النمويلية والادارية والتسويقية امام المشروعات الصغيرة

والمتوسطة.

المراجح

\section{أولاً، باللغة العربية}

يسري بطرس(1| • (Y)، "عوامل النمو الاقتصادي دراسة مقارنة بين مصر

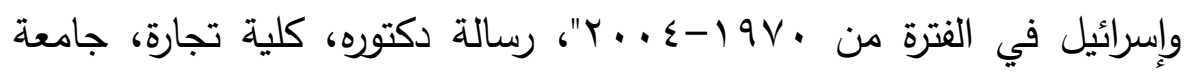

عين شمس.

روبرت صولو(ب . . r)، "نظرية النمو"، ترجمة ليلي عبود، المنظمة العربية

للترجمة، مركز دراسات الوحدة العربية، بيروت، لبنان.

ميشل تودارو(9 (9 ())، "التمية الاقتصادية"، ترجمة ومراجعة: محمودحسن

حسني، محمود حامد عبد الرازق( ( . . ب)، دار المريخ للنشر الرياض. 


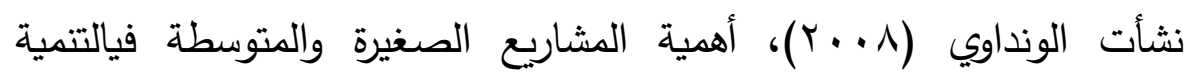
الاقتصادية وسبل النهوض بها في العراق، مجلة جامعة كربلاء العلمية، العدد

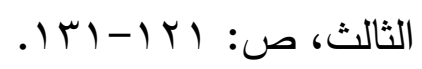

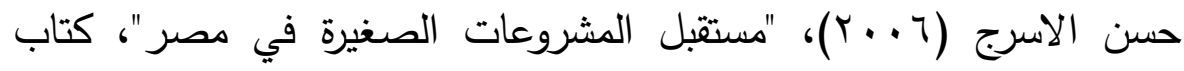

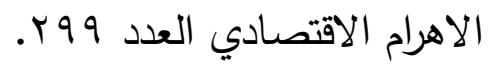

خياري ميرة (r ( • r)، "دور المؤسسات الصغيرة والمتوسطة في تحقيق التتمية الاقتصادية دراسة حالة المؤسسات الصغيرة والمتوسطة بولاية أم البواقي

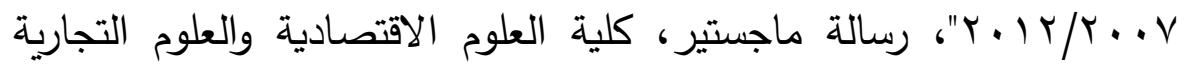
وعلوم التسيير، جامعة العربي بن مهيدي، أم البواقي، الجزائر . كفاح أبو ناجي (ع ( ب)، "الاثر التنموي للمشروعات الصغيرة الممولة من قبل وزارة الاقتصاد الوطني"، رسالة ماجستير، كلية التجارة، الجامعة الاسلامية، غزة، فلسطين.

\section{ثانيا، باللغة الانجليزيـة}

- Bello, A., et al., (2018), " Impact of Small and Medium Scale Enterprises on Economic Growth: Evidence from 
Nigeria", Global Journal of Economics and Business, Vol. 4, No. 2 , pp., $236-244$.

- Mare, D.C., (July 2004), "What Do Endogenous Growth Models Contribute?", Working Paper, Economic and Public Policy Research, 24390, PP: 1-30.

- Romer, P.M.( October 1986), "Increasing Returns and Long-Run Growth", Journal of political Economy,NO.5, PP:1002-1037.

- Romer, P.M., (October 1990), "Endogenous Technological Change", Journal of political Economy, NO.6, PP:98-130.

- Taiwo, M.A., et al. (2012), " Impact of Small and Medium Enterprises on Economic Growth and Development", American Journal of Business and Management, Vol. 1, No. 1, pp., 18-22.

- Thorsten, et al., (2003) "Small and Medium Enterprises Across The Global : aNew Database", World Bank Policy Research 